\title{
Corela
}

Cognition, représentation, langage

HS-31 | 2020

Métalinguistiques.

\section{Marqueurs discursifs du dire (français, russe, norvégien)}

Des sémantiques lexicales différentes pour des stratégies énonciatives similaires?

\section{Elizaveta Khachaturyan}

\section{(2) OpenEdition}

\section{Journals}

Édition électronique

URL : http://journals.openedition.org/corela/11158

DOI : $10.4000 /$ corela. 11158

ISSN : $1638-573 \mathrm{X}$

Éditeur

Cercle linguistique du Centre et de I'Ouest - CerLICO

\section{Référence électronique}

Elizaveta Khachaturyan, « Marqueurs discursifs du dire (français, russe, norvégien) », Corela [En ligne], HS-31 | 2020, mis en ligne le 05 juin 2020, consulté le 03 juillet 2020. URL : http://

journals.openedition.org/corela/11158; DOI : https://doi.org/10.4000/corela.11158

Ce document a été généré automatiquement le 3 juillet 2020

\section{cc) (†) ()}

Corela - cognition, représentation, langage est mis à disposition selon les termes de la licence Creative Commons Attribution - Pas d'Utilisation Commerciale - Partage dans les Mêmes Conditions 4.0 International. 


\section{Marqueurs discursifs du dire (français, russe, norvégien)}

Des sémantiques lexicales différentes pour des stratégies énonciatives similaires?

\section{Elizaveta Khachaturyan}

1 L'activité métalinguistique est une partie intégrante de l'activité langagière (indépendamment de la langue). Elle est souvent signalée dans le discours par des expressions et des marqueurs discursifs. Le point de départ de la présente analyse est une question de caractère formel: peut-on dire que, même s'il s'agit d'une activité universelle, les instruments à son service varient d'une langue à l'autre ? Cette question a été suscitée par une observation contrastive: dans certaines langues (comme en français et en russe analysés ici, mais aussi dans d'autres langues romanes, par exemple), les expressions métalinguistiques contiennent souvent une forme du verbe dire. Or, en norvégien les expressions avec le verbe å si ('dire') sont très peu fréquentes. Est-ce qu'on peut dire que le verbe dire (en général, utilisé pour décrire l'activité langagière et les problèmes de communication) n'est pas un élément obligatoire pour former les expressions signalant l'activité métalinguistique ? Pour répondre à cette question nous allons décrire quelques marqueurs discursifs (désormais MD) en français et en russe qui peuvent être définis comme métalinguistiques (en tant qu'éléments signalant l'activité langagière) et nous allons essayer de trouver leurs équivalents en norvégien.

2 La structure de l'article est la suivante. Dans la première partie sera présentée l'approche théorique dans laquelle nous travaillons ; dans la deuxième partie, les MD disons et skažem seront analysés pour illustrer leur rôle dans la construction du discours. Dans la troisième partie, nous analyserons les expressions norvégiennes qui signalent les mêmes types de problèmes de communication et sont employées dans des contextes proches. 


\section{Problèmes de communication. Le rôle des MD}

3 Pour décrire l'activité métalinguistique gérée par les MD nous allons utiliser la conception de la communication présentée dans les travaux d'A. Culioli (en particulier Culioli (1999) et (2018)). Cette conception est également à la base de la notion de 'scène énonciative' formulée dans les travaux de D. Paillard et utilisée pour décrire la sémantique discursive des MD.

D'après Culioli, l'activité de communication est basée sur l'ajustement entre deux sujets parlants. Cet ajustement est nécessaire car chacun possède sa représentation $R \mathrm{du}$ monde :

«Il faut donc bien comprendre que c'est aux deux bouts du schéma de communication qu'a lieu l'activité signifiante; le message n'a pas de sens, de contenu en soi, mais véhicule grâce à un enchaînement d'unités sonores ce qui a été $\mathrm{du}$ sens dans le langage intérieur du locuteur et redeviendra du sens dans le langage de l'auditeur » (Culioli 1999: 67).

Dans cette citation, « du sens dans le langage intérieur » correspond à la représentation qui a été celle du locuteur et redeviendra celle de l'interlocuteur.

6 L'ajustement qui est à la base de la communication suppose que, d'un côté, le locuteur essaye de transmettre à son interlocuteur sa représentation du monde à travers les mots. De l'autre côté, l'interlocuteur essaye d'accéder à ce qui est dit : « ...un second sujet qui va être amené à reconstruire et à rechercher éventuellement un ajustement avec ce que le premier sujet avait en tête » (entretien avec A. Culioli ; Ducard 2004: 10).

7 Ainsi, le procès de la communication peut être illustré par le schéma suivant :

$$
\text { So ( } \left.\mathrm{R}^{\prime}\right) \text { - mots - S1 (R) }
$$

où $\mathrm{S} 0$ est le locuteur - celui qui émet le message, dans la suite de notre analyse, ce sera celui qui émet le message avec la construction analysée -, S1 est l'auditeur (ou l'interlocuteur) - celui qui reçoit (et doit interpréter) le message émis. Chaque participant de la communication (S0 de même que S1) a sa propre représentation (R) du monde qu'il essaye de faire partager à son interlocuteur. $R$ et R' ne sont accessibles qu'à travers les mots qui sont échangés et le fait qu'il s'agisse des mêmes mots ne suffit pas à garantir que la représentation $\mathrm{R}$ déclenchée chez l'auditeur coïncide avec la représentation de départ $\mathrm{R}^{\prime}$ du locuteur, précisément dans la mesure où cela passe par des mots.

Les mots sont ce qui donne une forme à ce qui est à dire, ils constituent la matérialité du message par laquelle cet «à dire » devient accessible à S1. Or, les mots auxquels recourt le locuteur pour dire le monde ne constituent qu'une façon parmi d'autres de le dire. Il n'existe pas de rapport univoque entre les mots et le monde ou l'objet du monde (en fait, plus exactement, la représentation que s'en fait le locuteur) que ces mots sont censés dire. Comme le dit Culioli :

« les mots, ces médiateurs par excellence, nous font ressentir leur opacité et nous révèlent qu'il n'y a pas une relation immédiate et nécessaire entre les termes et les choses. Nous avons alors le sentiment que les mots nous trahissent, s'interposent entre nous et «l'indicible», cet «indicible » que nous n'arrivons pas à exprimer dans la chaîne sonore. » (1999: 65)

10 Ainsi, ce hiatus entre le projet du locuteur et les mots (et donc la propre évaluation de son dire par so) est en grande partie à l'origine de la difficulté de l'ajustement intersubjectif. Ce qui correspond au problème central de la communication reflété à sa 
manière par la formule emblématique : comment dire ? / kak skazat'? ('comment dire' en russe).

Cette insuffisance (et parfois incohérence) du dit explique l'impossibilité de dire de manière transparente et alimente une tendance à reformuler, commenter, dire plusieurs fois.

Une conception semblable de la communication a été proposée par les linguistes appartenant à d'autres écoles linguistiques. Par exemple, dans la théorie de la vagueness: "speakers must find a way to evoke appropriate mental representations of the people, places, and things they want to talk about. For any given entity, there is an unlimited variety of ways to refer to it, and candidate expressions in a language vary in their level of precision of reference " (Jucker et al. 2003: 1739). Le "appropriate mental representation" correspond à ce que nous appelons la représentation. Il y a un autre point important dans cette conception : "unlimited variety of ways " présuppose qu'il n'y a pas de mots exacts qui disent telle ou telle représentation, mais façon et façon de dire ce qui est à dire, car chaque sujet parlant possède son propre « code " (au sens qui lui est attribué par A. Culioli) : «nous possédons tous notre code à nous, qui ne correspond que partiellement à celui des autres. D'où des malentendus, un sentiment parfois douloureux d'incompréhension, le mur que dressent certaines maladies mentales... » (1999: 68).

Dans le discours, cet ajustement entre les interlocuteurs peut être signalé par différentes expressions. En français, par exemple, une formule emblématique de l'ajustement entre des sujets est la suivante: "Si tu vois ce que je veux dire par là ». Elle peut être interprétée de la façon suivante: "si tu vois [= si tu peux former ta représentation $\mathrm{R}]$ ce que je veux dire [R'] par là [= mots formant le message]». Il est intéressant que l'accessibilité du message est souvent liée à la visibilité du message.

trouve la même idée de rendre accessible, au sens de visible, à travers les mots dans la sémantique du verbe dire, telle qu'elle est décrite par J.J.Franckel (2016) : «Le propre de dire est d'introduire une articulation visible / invisible, ou non visible pour S. (...) C'est en raison de la mise en jeu de cette articulation visible / invisible qu'une assertion introduite par dire n'est jamais équivalente à l'assertion simple correspondante. Comparer par exemple : voici ce qui s'est passé / je vais vous dire ce qui s'est passé (dévoilement) » (2016: 134).

En russe également les verbes videt' ('voir') et skazat'1 ('dire') sont employés dans les expressions signalant des problèmes de communication et l'ajustement à venir, par exemple : vidiš' li ('vois-tu'), ja xotel skazat' sovsem drugoe ('je voulais dire tout autre chose'), etc.

A travers ces expressions qui expriment le hiatus entre le vouloir dire et le dire effectif, le locuteur essaye de signaler un problème qui s'est créé au cours de la communication ou de recevoir le feed-back de l'interlocuteur pour contrôler si la réception de la représentation transmise est réussie. Même si la forme de ces expressions peut varier d'une langue à l'autre, les éléments qui les constituent sont souvent les mêmes : ce sont, en particulier, les verbes voir/videt' et dire/skazat', dont la sémantique est liée à la mise en jeu du visible. Il faut remarquer que l'étymologie des verbes dire/skazat' renvoie au procès d'indication. A l'origine du verbe dire se trouve la racine deik/dik qui signifie 'montrer', 'indiquer'; on la trouve également dans le mot déictique et dans le verbe allemand zeigen ('montrer') (Dictionnaire étymologique Larousse). Dans le verbe skazat', le préfixe $s$ - est ajouté à la base -kaz- que l'on retrouve aussi dans les verbes préfixés : 
ukazat' (indiquer) et pokazat' (montrer) (Dobrušina \& Paillard 2002; Khachaturyan 2006).

17 Parmi les expressions signalant les problèmes de communication on peut distinguer un groupe d'éléments appelés «marqueurs discursifs du dire» (désormais MD du dire). Dans la section suivante, nous allons brièvement résumer en quoi consiste leur rôle en nous concentrant, en particulier, sur les marqueurs disons et skažem.

\section{La place des MD du dire dans la classification des MD}

\subsection{Classification des marqueurs discursifs}

Notre approche des MD s'inscrit dans le cadre théorique et méthodologique présenté dans les travaux de D. Paillard $(2009,2013)$. La base de cette approche est constituée par la Théorie des Opérations Prédicatives et Énonciatives d'A. Culioli. Dans le cadre de la méthodologie élaborée, les MD sont considérés en tant que éléments qui (comme toutes les autres parties du discours) se caractérisent par leur propre sémantique (explicitement indépendante de la pragmatique), désignée comme sémantique discursive, et par leurs propres propriétés distributionnelles. Pour définir la notion de 'sémantique discursive' la notion de 'scène énonciative' a été introduite par D. Paillard :

«la notion de scène énonciative repose sur l'hypothèse que l'énonciation n'est pas l'acte d'un sujet qui produit un énoncé mais un processus qui peut être reconstitué à partir de l'agencement des formes (...) qui composent un énoncé. L'énonciation est donc l'ensemble des déterminations (...) qui interviennent dans la production de l'énoncé ». (Paillard 2013: 157)

Dans cette perspective, les MD introduisent une détermination «spécifique portant sur telle ou telle composante de la scène énonciative" (ibid.). Six classes de MD ont été distinguées (Paillard \& Vu-Thi 2012) en fonction des déterminations de la scène énonciative signalées par le MD. Parmi ces six classes, une classe est constituée par les MD du dire : "la sémantique de ces unités porte sur le fait que l'énoncé (ou fragment d'énoncé correspondant à la portée du marqueur discursif) est la mise en mots d'un vouloir dire extérieur (vouloir dire d'un sujet, des mots ou du monde) "(Paillard 2009: 126). Ces marqueurs sont concentrés sur les mots $\mathrm{p}$ (la portée du marqueur) employés pour dire la représentation du monde, et signalent qu'il existe quelque problème dans le lien que le locuteur essaye d'établir entre les mots et ce qu'il a à dire.

Dans la description proposée dans Paillard \& Vu-Thi (2012), une propriété importante des MD du dire est leur forme : parmi les exemples cités, on ne retrouve que des MD contenant le verbe dire : disons, pour ainsi dire, soi-disant, je voulais dire, c'est-à-dire. Par la suite nous allons essayer de voir si la présence du verbe dire est une condition obligatoire pour cette classe de MD, et si la même sémantique discursive peut être mise en jeu par des marqueurs qui ont une forme différente dans une autre langue. Dans la présente analyse, la question sera posée à propos du norvégien.

Pour répondre à cette question, essayons, d'abord, de voir en quoi consiste la spécificité des MD du dire, spécificité qui dans la classification proposée dans Paillard \& Vu-Thi (2012) est étroitement liée à la sémantique des verbes dire et skazat'. 


\subsection{Les MD du dire}

du schéma de communication. La sémantique de dire prend en compte le rapport entre les mots et S0 qui utilise les mots pour "extérioriser " (ou rendre visible, d'après Franckel) ce qu'il a à dire (sa représentation). La sémantique de skazat' est focalisée non seulement sur l'extériorisation mais surtout sur l'ajustement intersubjectif : elle est centrée sur les figures de S0 et S1, marquant que les mots de So déclenchent une représentation dont le support est $\mathrm{S}^{2}$. Cette différence a des conséquences pour la sémantique des MD du dire dans les deux langues ${ }^{3}$. La sémantique des MD du dire avec skazat' (en particulier, de skažem et de tak skazat' 'ainsi dire') est focalisée sur les rapports entre $\mathrm{S} 0$ et $\mathrm{S} 1$. La sémantique des $\mathrm{MD}$ du dire avec le verbe français dire (disons et pour ainsi dire) met en avant le rapport existant entre $\mathrm{S} 0$ et le dit. Ces différences dans la sémantique expliquent les propriétés distributionnelles de ces $\mathrm{MD}^{4}$. Le MD skažem est très fréquent dans un discours dialogique, où le locuteur prend en compte et s'adresse à l'interlocuteur réel et actif qui peut vraiment contribuer à la validation du terme $p$ employé. Le MD disons est souvent employé dans le discours monologique où le locuteur doit faire face à l'insuffisance des mots.

Malgré ces différences, skažem et disons peuvent être employés dans les contextes similaires, qui sont souvent définis comme exemplification, précision, stipulation d'un accord (Franckel 2016; Khachaturyan 2006 ; Paillard \& Vu-Thi 2012). Par exemple : (a) On se voit, disons demain; (b) Možet byt' poobedaem vmeste? Ja priglašaju. Skažem zavtra. ('Peut être pourrions-nous déjeuner ensemble ? C'est moi qui vous invite. Skažem demain.')

En même temps, chacun de ces marqueurs a ses emplois typiques à travers lesquels les différences sémantiques peuvent être illustrées. Dans le cas de disons, le locuteur recourt à une dénomination, marquée comme en suspens, mais qui, en réalité, ne doit pas être stabilisée dans la mesure où $\mathrm{p}$ est une partie du projet (de l'énoncé) appartenant au locuteur et visant à dire ce qu'il a à dire. Dans (1), la séquence disons $p$ relève d'une dénomination arbitraire / conventionnelle :

(1) En temps de paix, dans un Etat organisé, avec une hiérarchie qui est formée pour respecter l'Etat, je crois que chaque fonctionnaire a le devoir d'obéir. Dans une période, disons révolutionnaire, quand l'Etat n'était pas maître de son sort, comme c'était le cas sous l'Occupation, il en est tout autrement, et j'aurai l'occasion d'en reparler. (Le Monde)

Le terme $\mathrm{p}$ (révolutionnaire) est employé pour désigner une période historique, qui $a$ priori n'est pas définie en tant que telle, mais qui est opposée à une autre période définie dans le contexte précédent comme temps de paix. Dans le contexte droit, so, pour préciser ce qu'il entend par révolutionnaire, recourt à une description explicite : phrase subordonnée - quand l'Etat n'était pas maître de son sort.

Skažem est focalisé sur les interlocuteurs : $\mathrm{p}$ est une dénomination vague à laquelle recourt le locuteur pour dénommer ce qu'il a à dire sans en assumer la responsabilité. Mais il n'assume pas la responsabilité de la représentation que $\mathrm{p}$ peut déclencher et le laisse interpréter à $\mathrm{S} 1, \mathrm{p}$ étant une expression trop vague. Dans le discours dialogique, il s'agit souvent d'une vraie dérobade (comme dans (2)): l'interlocuteur pose une question à laquelle le locuteur ne veut pas répondre en assumant la responsabilité de la réponse ; le locuteur se dérobe en donnant une réponse sous toute réserve.

Corela, HS-31 | 2020 
(2) A kto nočuet na vokzale? - Griša zamjalsja. - Eto nevažno. Nu, skažem ... ja. (Lazarčuk)

'- Mais qui dort à la gare? - Griša hésita : - Ça n'a pas d'importance. Eh bien, skažem ... moi.'

Ce type ne relève pas uniquement d'emplois dialogiques où le locuteur et l'interlocuteur sont les figures réelles. Le recours à S1 en tant que support d'une représentation peut correspondre à la prise en compte de la représentation qui peut être évoquée par p mais a priori n'est pas prévue par le développement de la narration. Dans ce cas, $p$ correspond à une dénomination très vague, souvent une formule :

(3) Ob idealah: ty rodilsja $\mathrm{v}$ strane kommunističeskih idealov, bežal ot nih $\mathrm{k}$ sionistskim idealam, a potom, ešče bystree $\mathrm{k}$ idealam... nu, skažem, svobody $\mathrm{i}$ demokratii. No uže v tret'ej iz tvoih stran mestnye idealisty gotovy povesit' tebja na pervom podvernuvšemsja stolbe. (Reznik)

'A propos des idéaux : tu es né dans le pays des idéaux communistes, ensuite tu as fui vers les idéaux sionistes, et ensuite, encore plus vite vers les idéaux ... eh bien, skažem de la liberté et de la démocratie. Mais voilà que déjà, dès le troisième de ces pays, les idéalistes locaux sont prêts à te pendre au premier poteau venu.'

\section{Correspondances en norvégien}

\subsection{Les expressions avec å si ('dire')}

À première vue, les expressions avec le verbe dire (å si) employées pour signaler l'activité métalinguistique sont très rares en norvégien. Pour vérifier cette première impression nous avons analysé deux types de corpus: un corpus de norvégien écrit (Oslo-korpus ${ }^{5}$ ) et un corpus de norvégien oral $\left(\mathrm{NoTa}^{6}\right)$. L'équivalent ${ }^{7}$ de disons et skažem pourrait être la construction la oss si ou le verbe si (forme à l'infinitif) est employé avec la forme à l'impératif du verbe å la ('laisser', 'to let' en anglais) et le pronom à la $1^{\mathrm{e}}$ pers. pl. (oss, 'us' en anglais). A priori la construction la oss accompagnant un verbe correspond à l'injonction de faire ensemble. Parmi les autres équivalents possibles de disons et skažem, on peut distinguer les constructions avec le pronom à la $1^{\mathrm{e}}$ pers.pl. - vi ('nous') - et les verbes modaux (VM) : kan ('pouvoir', forme conjuguée), skal ('devoir/ pouvoir', forme conjuguée ; cette forme verbale est employée pour former le futur), må ('devoir', forme conjuguée). Voyons ci-dessous comment ces constructions sont employées et distribuées dans les deux corpus analysés.

\subsubsection{La oss si}

La construction la oss si est extrêmement peu fréquente dans les deux corpus : moins de 30 occurrences dans chaque corpus. En se basant sur les propriétés formelles (le contexte d'emploi et la forme de la portée), on peut distinguer les cas d'emploi suivants.

1) Dans la plupart des exemples, la construction la oss si introduit une phrase subordonnée accompagnée par les formes at ('que') ou hvis ('si') :

(4) På spørsmål om hvor mye avtalen kostet, svarte han : - La oss si at den kostet en hel del (Oslo-korpus)

'A la question concernant le prix du tarif, il répond : - La oss si que cela a coûté très cher.'

(5) Nja... vi kan ta Jonas som eksempel. La oss si at kroppen hans består av energilegemer bygd opp av biologisk plasma. (Oslo-korpus) 
'Eh, oui. Nous pouvons prendre Jonas comme exemple. La oss si que son corps contient des organismes composés de plasma biologique.'

(6) ... men la oss si at vi drar til Stockholm da må vi jo \# finne et sted å bo. (NoTakorpus)

'... alors, la oss si que nous allons à Stockholm, alors nous devons \# trouver une place où dormir.'

Dans (4), il s'agit d'une réponse à une question ; dans (5) et (6), toute la construction introduit un exemple.

2) Dans quelques rares exemples, la oss si est employé avec la forme du pronom det ('cela') qui du point de vue syntaxique correspond au complément d'objet direct.

(7) Jeg har alltid dikta historier. Skrevet og fortalt. Og jugdt veldig mye. - Også som liten? - La oss si det sånn: jeg var en fantasifull unge. (Oslo-korpus)

'J'ai toujours inventé des histoires. Je les écrivais et racontais. Et je mentais beaucoup. - Quand tu étais petit ? - La oss si det ('cela') sånn ('comme ça') : j'ai été un garçon très créatif.'

Dans (7), la construction analysée introduit la réponse à une question.

On voit que dans le cas de la réponse à la question (ex. 4 et 7), le locuteur essaie de donner une réponse quelque peu vague sans en assumer la responsabilité (comme c'était le cas dans les emplois avec skažem).

3) Dans une dizaine d'exemples, la oss si introduit une portée correspondant à un mot ou à un syntagme. Cette portée a très souvent la forme des numéros ou des noms propres, qui ne peuvent pas avoir plusieurs interprétations, mais sont employés pour donner un exemple.

(8) Hvis vi forsøker å finne ut hvor mange jungeltelegrafen hadde nådd frem til før la oss si klokken kvart på tolv, og setter opp en navneliste ...(Oslo-korpus)

'Si nous essayons de savoir combien de personnes ont été informées avant la oss si midi moins le quart, et de préparer une liste avec des noms ...'

(9) ... hvis jeg går ut og trener la oss si på Sats eller på noe sånn men \#\# på skolen ... (NoTa-korpus)

'... si je sors et je vais à la gym la oss si à Sats ${ }^{8}$ ou quelque chose comme ça mais \# à l'école ...'

Dans le corpus parlé, la oss si est employé dans le cas où le locuteur se trouve vraiment en difficulté pour trouver 'quoi dire'. Cette hésitation du locuteur peut être marquée par d'autres éléments : pauses, ajout de eller ('ou'), emploi de liksom ('genre' - 'like' en anglais).

4) Dans un seul exemple que nous avons trouvé dans Oslo-korpus, la portée de la oss si peut être interprétée en tant que terme choisi sous toute réserve :

(10) Men ingen vil ha meg med. Vi vet jo hvordan vår politikk er - la oss si uspiselig

- og er villige til å inngå kompromisser. (Oslo-korpus)

'Mais personne ne me veut. On sait bien comment notre politique est - la oss si peu gentille - et on est prêt à faire des compromis.'

Uspiselig peut signifier 'non sympathique', quand il s'agit d'une personne. Ce n'est pas un terme tout à fait adéquat, quand on parle des propriétés de la politique.

\subsubsection{Verbes modaux + vi si}

Les constructions avec le verbe modal sont aussi très rares dans les deux corpus (moins de 100 exemples au total). Parmi ces exemples, on peut distinguer deux groupes d'emplois. 
40 1) Dans un premier groupe (le plus nombreux), la construction verbe modal + vi si est centrée sur l'action même de dire: dans le contexte, les raisons ou le temps sont spécifiés (som en konklusjon 'en conclusion', oppsummeringshvis 'en résumé', derfor 'donc', dermed 'ainsi', generelt 'en général', etterpå 'après', nå 'maintenant', etc.), une phrase subordonnée est introduite après. Il s'agit, en général, de la construction avec kan ('pouvoir') :

(11) Generelt kan vi si at de anti-klassiske nordiske former forutsetter de klassiske.

(Oslo-korpus)

‘En général kan vi si (= nous pouvons dire en général) que les formes nordiques

anti-classiques impliquent la présence des formes classiques.'

41 A notre avis, dans ce cas il ne s'agit pas d'un emploi discursif car aucun statut discursif n'est transmis à ce qui est dit. On ne peut pas dire, en suivant D. Paillard, que ces expressions indiquent «que la séquence correspondant à leur portée relève d'un vouloir dire autonome distinct du vouloir + dire du locuteur ${ }^{9} »($ Paillard 2009: 124).

2) Dans un autre groupe (moins nombreux) d'exemples, la construction verbe modal + vi si est centrée sur les mots qui font partie de la portée de la construction. Cela peut être le cas que l'on peut définir comme " exemple » comparable avec les emplois de disons et skažem (ex. a et b dans la section 2.) :

(12) Du kommer hjem til meg i morgen kveld. Skal vi si ved halv åtte-tiden ? (Oslokorpus)

'Tu viens chez moi demain soir ? Skal vi si vers sept heures et demi.'

Dans un autre type d'emploi (ex. 13 et 14), la construction avec le verbe si signale la recherche de terme adéquat. On peut remarquer que dans le contexte précédent il y a souvent un autre terme qui a été proposé pour dire le même état de chose et on a la conjonction eller ('ou').

(13) Deres ferd gjennom Sahara vil bli en lysttur, eller skal vi si over Sahara? Hehe.

Flyet går en gang i uken. (Oslo-korpus)

'Votre voyage à travers le Sahara deviendra un voyage d'agrément, ou skal vi si audessus du Sahara ? Hehe. L'avion part une fois par semaine.'

(14) ... de motsetter seg mot en slik ekstra søppelavgift - eller skal vi si ekstra søppelskatt ...(Osla-korpus)

'... ils s'opposent à l'introduction de ce type de charge supplémentaire sur les déchets ou skal vi si à un impôt supplémentaire sur les déchets ...'

L'emploi dans (13) et (14) peut être interprété comme renvoyant à un ajustement à venir entre les interlocuteurs : le locuteur se trouve devant le choix d'un terme adéquat et l'interlocuteur peut y être impliqué.

A l'oral, d'autres expressions signalant la recherche d'un mot en cours peuvent précéder la construction avec si (comme $e$ et la répétition de blitt dans l'exemple (15) cidessous) :

(15) ... som på en måte har blitt e \#\# blitt e skal vi si levebrødet \# senere så at jeg \# jeg jobber jo innenfor underholdning (NoTa)

'... ce qui d'une certaine façon est devenu eh \#\# devenu eh skal vi si mon pain quotidien \# plus tard donc je \# je travaille donc dans le divertissement.'

Tous les cas d'emplois des expressions avec le verbe å si ('dire') présentés ci-dessus, malgré un fonctionnement qui peut être considéré comme comparable avec les MD disons et skažem, sont très peu fréquents. Existe-t-il d'autres expressions qui ont en norvégien le même rôle que les MD du dire en français et en russe? 


\subsection{Les expressions avec les verbes å hete ('appeler'), å kalle ('appeler')}

Parmi ces expressions, on trouve des constructions utilisant non pas le verbe å si ('dire'), mais deux verbes que l'on peut traduire en français par le verbe 'appeler' : $a$ hete, å kalle. Ces deux verbes peuvent marquer la prise en compte de l'interlocuteur, mais de deux façons différentes. Le verbe å hete renvoie à l'idée 'avoir comme nom' et implique un savoir partagé. Par exemple : jeg heter Petter ('je m'appelle Petter'), han har ikke smakt det som heter alkohol på tre måneder ('il n'a pas gouté ce qui s'appelle de l'alcool pendant trois mois') ${ }^{10}$. Le verbe å kalle met en jeu le procès de 'nommer / donner le nom', cette action renvoyant à la recherche d'un nom adéquat effectuée ensemble. Par exemple: Vi kaller henne sjefen. ('Nous l'appelons notre chef'), Kaller du det å vaske? (‘ Tu appelles cela laver ?'). On peut dire que les deux verbes sont concentrés sur les problèmes de nomination. L'acte de nomination peut comporter le recours à un terme arbitraire choisi provisoirement ou sous toute réserve. En outre, la nomination prévoit une sorte d'ajustement, car le nom donné doit être accepté ou partagé (au moins provisoirement) par l'interlocuteur. Comme nous avons vu, ces problèmes sont aussi mis en jeu dans les emplois de disons ou skažem. Par la suite nous allons décrire les cas d'emplois des expressions norvégiennes.

\subsubsection{Les expressions avec å hete}

Les expressions avec le verbe å hete sont plus fréquentes : elles peuvent avoir la forme d'une question (hva heter det 'comment ça s'appelle') ou d'une phrase subordonnée (som heter 'qui s'appelle'). Ces expressions sont employées surtout à l'oral où elles sont accompagnées par des pauses ou par d'autres marques d'hésitation, comme jeg husker ikke ('je ne me souviens pas'), eller ('ou'). Un autre terme n'est souvent même pas introduit, et la phrase est laissée ouverte à l'introduction d'autres noms possibles.

(16) Jeg vet ikke de fikk jo også \#\# hva heter det da\#\# tilbud om å flytte. (NoTa)

'Je ne sais pas ils ont aussi reçu \#\# comment ça s'appelle \#\# offre de déménager.'

(17) Jeg blir sånn innen høgskole idrettslærer eller hva det nå heter da ... (NoTa)

'Comme ça je suis devenu dans une école supérieure prof de gym ou comment ça

s'appelle maintenant ...'

Il faut remarquer que dans le contexte qui précède l'expression avec le verbe hete les éléments suivants sont souvent présents :

pronom indéfini noe ('quelque chose') qui introduit l'objet pour lequel le nom est recherché :

(18) Så gikk jeg på noe som heter e framhaldsskole ... (NoTa)

'Je suis allé dans quelque chose qui s'appelle eh école secondaire ...'

un rejet explicite d'un terme employé avant :

(19) ... rulleskøyter nei hva heter det skrunøkkelskøyter ... (NoTa)

'... patins à roues alignées non comment ça s'appelle patins à clé ...'

Il faut remarquer que dans les deux exemples ci-dessus (18 et 19) le terme (p) introduit par l'expression avec hete peut être considéré comme un terme en suspens dont le vouloir dire ne correspond pas parfaitement à ce que le locuteur veut dire, et la collaboration de l'interlocuteur peut être utile dans ce procès de nomination ou d'acceptation provisoire du terme. Dans (18), le mot framhaldsskole est signalé par le dictionnaire comme terme vieilli. Dans (19), le mot skrunøkkelskøyter n'existe pas dans le 
dictionnaire, mais le mot est facilement décomposable en deux parties : skrunøkkel ('clé de serrage') et skøyter ('patins') et on comprend sans difficulté qu'il s'agit des patins qui s'attachent aux bottes avec une clé et qui sont appelés d'habitude skruskøyter tout court.

\subsubsection{Les expressions avec å kalle}

Le terme $\mathrm{p}$ introduit par l'expression avec le verbe kalle se présente comme point de négociation entre les interlocuteurs, sur le choix duquel l'interlocuteur peut intervenir. Ce recours à l'interlocuteur est souvent marqué par la forme même de l'expression: cela peut être une question qui semble presque attendre l'approbation de l'interlocuteur (comme dans l'exemple (20)) ou cela se marque par la forme de $1^{\mathrm{e}}$ pers. pluriel (comme dans (21)). Dans les deux exemples ci-dessous, le terme p peut être interprété comme un terme sous réserve qui n'est pas parfaitement adéquat pour dire ce que le locuteur a à dire.

(20)... på en måte er det veldig greit å bo der men e litt av problemet der og er jo det med \#\# skal jeg kalle det da ? infrastrukturen og tilbud i forhold til skole og sånn. (NoTa)

'... dans un sens, il est très agréable d'y rester, mais il y a quelques problèmes là-bas avec dois-je l'appeler l'infrastructure et l'offre par rapport à l'école, quelque chose comme ça.'

(21) Der var det en sånn \# slags\# hva skal vi kalle det a en \# gressbakke av noe slag. (NoTa)

'C'était une cela \# une sorte \# comment nous allons l'appeler un ... une pente herbeuse en quelque sorte.'

Dans (20), infrastrukturen ('infrastructure') est un terme assez vague, et il y a une tentative pour le préciser par la suite: tilbud $i$ forhold til skole ('l'offre par rapport à l'école') peut être vue comme une sorte de précision. Mais à la fin le locuteur laisse la liste ouverte: og sånn (une formule parlée que l'on pourrait traduire par 'so on' en anglais, ou par 'quelque chose comme ça' en français).

Il faut remarquer que dans les exemples (20) et (21), d'autres expressions signalant l'insuffisance (ou l'inadéquation) du terme choisi sont employées: le mot slags ('une sorte de'), av noe slag ('en quelque sorte').

\section{Conclusion}

Ainsi, nous avons observé que les marqueurs et les expressions signalant l'activité métalinguistique ne sont pas toujours formés à partir du verbe dire. En norvégien, dans les expressions plus fréquentes, les formes des verbes 'appeler' - å hete et å kalle - sont utilisées. Tout en ayant une autre forme, ces expressions mettent en jeu des problèmes très similaires : la recherche d'un terme adéquat pour parler du monde. Cependant, le problème d'adéquation du terme choisi est vu différemment dans ce cas, dans la mesure où il s'agit de sémantique de la nomination, alors que pour la sémantique des MD du dire (en français et en russe) l'idée de la mise en mot (extériorisation) d'un vouloir dire du locuteur (sa représentation interne) est importante. Ainsi, le problème évoqué par les MD du dire est focalisé sur l'insuffisance des mots. Les expressions avec les verbes de nomination renvoient à un autre procès, celui de la nomination, donc de la recherche d'un mot qui peut être provisoirement ou conventionnellement adéquat pour parler du monde. 
nous reste, pourtant, quelques questions qui exigent une analyse ultérieure. La prévalence des verbes de nomination (en norvégien) peut-elle avoir quelques conséquences pour la construction du discours en général ? Existe-t-il d'autres moyens qui peuvent signaler l'insuffisance des mots (comme le font les MD du dire) ? Quel est le rôle des verbes de nomination en français et en russe par rapport aux expressions norvégiennes avec les mêmes verbes?

\section{BIBLIOGRAPHIE}

Authier-Revuz J. (1995). Ces mots qui ne vont pas de soi : boucles réflexives et non-coïncidences du dire. Paris : Larousse.

Culioli A. (1999). La communication verbale. Document ronéoté. UFRL. Université Paris 7. 65-73.

Culioli A. (2018). “Heureusement!”. Pour une linguistique de l'énonciation. Tours et détours.

Tome IV. Limoges : Lambert Lucas. 127-136.

Dobrušina E. \& Paillard D. (2001). Semantičeskie mexanizmy vzaimodejstvija pristavki i glagol'noj osnovy (osnova KAZ) (Les mécanismes de l'interaction préfixe - base verbale. Etude de la base kaz-). In Mengel S. (Hrsg), Actes de la 5ème conférence de dérivation lexicale des langues slaves (Halle, septembre, 2001). Slavische Wortbildung : Semantik und Kombinatorik. 263-280.

Ducard D. (2004). Entre grammaire et sens. Etudes sémiologiques et linguistiques. Paris : Ophrys.

Franckel J.J. (2016). Formes impératives de dire : disons, dis, dites et leurs variantes. In Rouanne L. \& Anscombre J.C. (eds). Histoire de dire. Petit glossaire des marqueurs formés sur le verbe dire. Peter Lang. 131-154.

Jucker A. H., Smith S. W., Lüdge T. (2003). Interactive aspects of vagueness in conversation. Journal of Pragmatics 35. 1737-1769.

Khachaturyan E. (2006). Les mots du discours formés à partir des verbes dire / skazat' en français et en russe, Thèse de doctorat en sciences du langage, Université Paris Diderot.

Khachaturyan E. (2010). The Function of Mitigation in Spoken Language. Analysis of tak skazat' (Russian) and diciamo (Italian). Oslo Studies in Language (OSLa). 393-412.

Khachaturyan E. (2013a). La sémantique des marqueurs discursifs du dire vue à travers la sémantique verbale. L'analyse des verbes dire/dire en français et en italien. In Casanova Herrero E., Calvo Rigual C. (eds). Actes del 26é Congrés de Lingüística i Filologia Romàniques (València, 6-11 de setembre de 2010). Berlin: W. de Gruyter. 207-221.

Khachaturyan E. (2013b). L'emploi du verbe dire avec la négation. L'étude contrastive des Constructions ne skažu, ne govorju, non dico en russe et en italien. In La linguistique de la contradiction. Peter Lang Publishing Group. 263-278.

Paillard D. (2009). Prise en charge, commitment, ou scène énonciative. Langue française 162. 109-128.

Paillard D. (2013). Les marqueurs discursifs comme catégorie In Dufaye L. \& Gournay L. (eds). Benveniste après un demi-siècle. Regarde sur l'énonciation aujourd'hui. Paris : Ophrys. 154-181. 
Paillard D. \& Vu-Thi N. (2012). Inventaire raisonné des marqueurs discursifs du français. Description. Comparaison. Didactique. Presse de l'Université Nationale de Hanoï.

\section{NOTES}

1. Faute de place, nous ne traitons pas ici la paire aspectuelle de skazat' - le verbe govorit' qui est aussi souvent employé dans les expressions métalinguistiques. (Khachaturyan 2013b)

2. Ce qui est lié également avec la forme préfixée du verbe: le préfixe $s^{-+}$la base -kaz(Khachaturyan 2006, 2013b).

3. Faute de place nous ne parlerons pas ici de la forme morphologique des deux MD analysés (disons et skažem) $-1^{\mathrm{e}}$ pers. pl. - qui peut être considérée comme un point en commun entre ces deux MD (p.ex. Authier-Revuz (1996)).

4. Par exemple, tak skazat' ('ainsi dire') est devenu un ponctuant de discours en russe, alors que pour ainsi dire est plus typique d'un registre plus soutenu (Khachaturyan 2006, 2010).

5. Ce corpus de 18,5 million de mots contient des textes pris dans des journaux, revues, romans, et documents publics.

6. Le corpus du norvégien parlé - NoTa - a été audio- et vidéo-enregistré en 2004-2006 dans la région d'Oslo avec 144 informateurs. Il contient environ 900000 mots transcrits.

7. Pour les objectifs de cet article nous allons nous concentrer uniquement sur les équivalent de disons et skažem, sans prendre en considération d'autres emplois de ce verbe.

8. Le nom d'une salle de sport à Oslo.

9. Il faut mentionner le fait que pour Paillard il existe deux types de vouloir dire : le vouloir dire 'projet' (« où le verbe dire commute avec n'importe quel autre verbe : c'est l'intention du locuteur qui est en jeu » (Paillard 2009: 114)) et le vouloir dire 'contenu' noté par «vouloir + dire » et désigné par le mot dire pris comme substantif (ibid.). Le vouloir + dire peut être « redéfini comme l'association d'un vouloir + dire et des formes qui composent l'énoncé » (ibid.).

10. Cet exemple et les exemples avec le verbe å kalle sont pris du dictionnaire Bokmålsordboka.

\section{RÉSUMÉS}

L'activité de communication est basée sur l'ajustement entre deux sujets parlants car «il n'y a pas une relation immédiate et nécessaire entre les termes et les choses » (Culioli). L'indicible que nous n'arrivons pas à exprimer et l'insuffisance du dit alimentent une tendance à reformuler, commenter, dire plusieurs fois. Traditionnellement on dit que les marqueurs discursifs du dire sont parmi les expressions les plus employées pour signaler l'activité métalinguistique. Leur rôle consiste "à mettre en scène un vouloir dire qui n'est pas celui du locuteur» (Paillard). La sémantique de ces marqueurs en français et en russe est liée à la sémantique des verbes dire et skazat' ('dire'). Par exemple, les différences entre skažem et disons peuvent être expliquées à travers les différences dans la sémantique des verbes. Mais que se passe-t-il en norvégien, où, comme nous verrons, les expressions avec le verbe $\stackrel{a}{a} s i$ ('dire') sont très peu fréquentes ? Nous analyserons les constructions avec les verbes 'appeler' ( $a ̊$ kalle, å hete) qui mettent en jeu des problèmes très similaires. 
The communicative activity is based on the adjustment between two speakers because the relationship between words and things is not immediate and obligatory (Culioli). In what is said there is always the indicible ('impossible to say') that we don't manage to express, and the words seem to be insufficient. The speaker tries to reformulate, to explain or to say in a different way, and the discourse markers 'du dire' (of the verb 'say') are often used in this case to show that what is said does not belong to the speaker (Paillard). The semantics of these discourse markers in French and Russian (i.e., disons and skažem) can be explained through the semantics of the verbs dire and skazat'. But what happens in Norwegian, where the expressions with the verb a si are not very frequent? We will analyze the expressions with the verbs å kale / a hete ('to call') that are focused on similar problems.

\section{INDEX}

Mots-clés : verba dicendi, sémantique verbale, schéma de communication, vouloir dire

Keywords : verba dicendi, verbal semantics, communicative model

\section{AUTEUR}

\section{ELIZAVETA KHACHATURYAN}

Université d'Oslo, Norvège 